\title{
Quantum harmonic oscillator fluorescence
}

Daniel Boye, Laurence Cain, Mario Belloni, Sarah Friedensen, Nancy Pruett, et al.

Daniel M. Boye, Laurence Cain, Mario Belloni, Sarah Friedensen, Nancy Pruett, Henry Brooks, "Quantum harmonic oscillator fluorescence," Proc. SPIE 11143, Fifteenth Conference on Education and Training in Optics and Photonics: ETOP 2019, 111430U (2 July 2019); doi: 10.1117/12.2523597 Photonics: ETOP 2019, 2019, Quebec City, Quebec, Canada 


\title{
Quantum harmonic oscillator fluorescence
}

\author{
Daniel M. Boye*, Laurence Cain, Mario Belloni, Sarah Friedensen, Nancy Pruett, Henry Brooks \\ Davidson College Physics Department, Davidson, NC 28035
}

\begin{abstract}
Although the Quantum Harmonic Oscillator (QHO) is examined with differing levels of rigor in physics and quantum optics, experiments exploring the QHO have not found broad use in classroom demonstration or laboratory instruction. We present a QHO exercise that is rock solid, inexpensive and easily implemented. The QHO system studied is the sulfur anion $\left(\mathrm{S}_{2}\right)^{-}$, an activator found in over a dozen minerals worldwide. A violet laser illuminates the sample immersed in liquid nitrogen and spectra containing lines evenly spaced in energy are collected. The energy spacing and effective spring constant for the electronic ground state QHO levels are determined. A close examination of the energy difference between consecutive levels reveals anharmonicity in the ground state potential.
\end{abstract}

Keywords: quantum harmonic oscillator, sulfur fluorescence, mineral fluorescence, diatomic molecule, quantum optics education

\section{INFORMATION}

The mathematical treatment of the Quantum Harmonic Oscillator (QHO) problem can be found in many optics and physics textbooks, with conceptual presentations even at the introductory level. Vibrational spectra of diatomic gases are often shown as illustrations of the evenly-spaced energy levels produced by a quadratic potential. However, providing opportunities for students to perform measurements on these gases is widely practiced in the teaching laboratory. This conference paper for the 2019 Education and Training in Optics and Photonics (ETOP) Conference presents instructions for an undergraduate level laboratory exercise that can be cast at the level of sophistication of the course and equipment available. The setup can also be adapted for classroom demonstration.

The QHO system studied is the sulfur anion $\left(\mathrm{S}_{2}\right)^{-}$, an activator found in over a dozen minerals worldwide. We present here two of those minerals. The family of natural minerals called scapolites is represented by the general formula $(\mathrm{Na}, \mathrm{Ca})_{4} \mathrm{Al}_{3}(\mathrm{Al}, \mathrm{Si})_{3} \mathrm{Si}_{6} \mathrm{O}_{24}\left(\mathrm{Cl}_{1} \mathrm{CO}_{3}\right)$ with varying relative amounts of the ions within the parentheses. The ideal formula for natural sodalite is $\mathrm{Na}_{8} \mathrm{Al}_{6} \mathrm{Si}_{6} \mathrm{O}_{24} \mathrm{Cl}_{2}$. The sulfur anion $\left(\mathrm{S}_{2}\right)^{-}$is one of several ions that can be contained within the alumino-silicate cages of these minerals. Other ions include $\mathrm{Na}^{+}, \mathrm{Ca}^{2+}, \mathrm{Cl}^{-}, \mathrm{CO}_{3}{ }^{2-}, \mathrm{SO}_{3}{ }^{2-}$, and $\mathrm{SO}_{4}{ }^{2-}$. Detailed examinations of the fluorescence from $\left(\mathrm{S}_{2}\right)^{-}$centers in these minerals can be found in References [1] and [2].

Scapolites with strong yellow emission can be found in Ward's Science fluorescent mineral collections containing "longwave" samples. A wide variety of minerals from around the world can be easily obtained by contacting Mark Cole (markcole@minershop.com) at the Minershop website (http://www.minershop.com/).

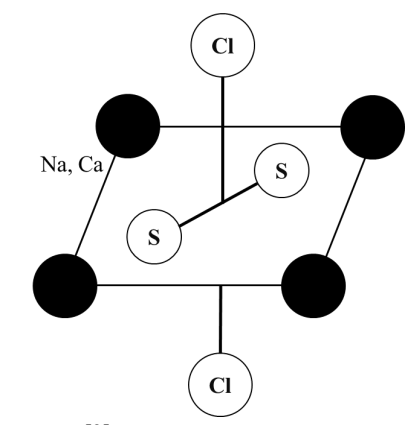

Figure 1. $\left(\mathrm{S}_{2}\right)^{-}$in an alumino-silicate cage. ${ }^{[3]}$

One configuration of $\left(\mathrm{S}_{2}\right)^{-}$in an alumino-silicate cage is shown in Figure 1. There are many possible configurations of ions surrounding the $\left(\mathrm{S}_{2}\right)^{-}$centers that will occur in a natural mineral. In addition, the host alumino-silicate structure 
may also contain impurities and imperfections. The resulting variations of the electric field strength and symmetry local to each $\left(\mathrm{S}_{2}\right)^{-}$gives rise to inhomogeneous broadening of the spectral lines that is present at liquid helium temperatures. There are only slight changes in the spectral profile for temperatures below $77 \mathrm{~K}$. Above $77 \mathrm{~K}$, the individual lines become less distinct since the optically-active electron of the $\left(\mathrm{S}_{2}\right)^{-}$center is exposed to thermal oscillations giving rise to local modulations in the electronic transition energy in the host material as shown in Figure 2.
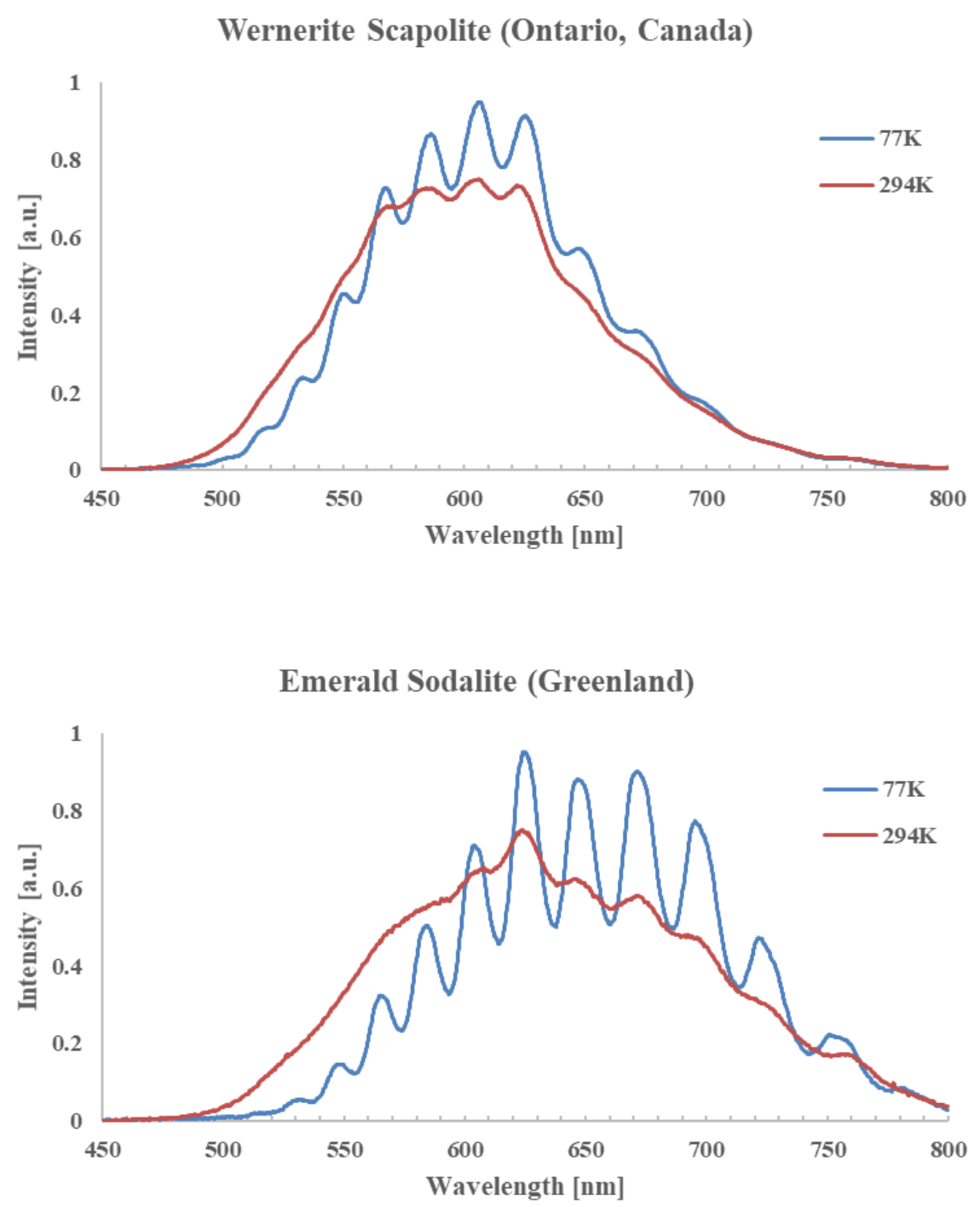

Figure 2. Fluorescence emission spectra for the indicated minerals.

A close examination of the energy difference between consecutive levels reveals anharmonicity in the ground state potential as shown in Figure 3. 

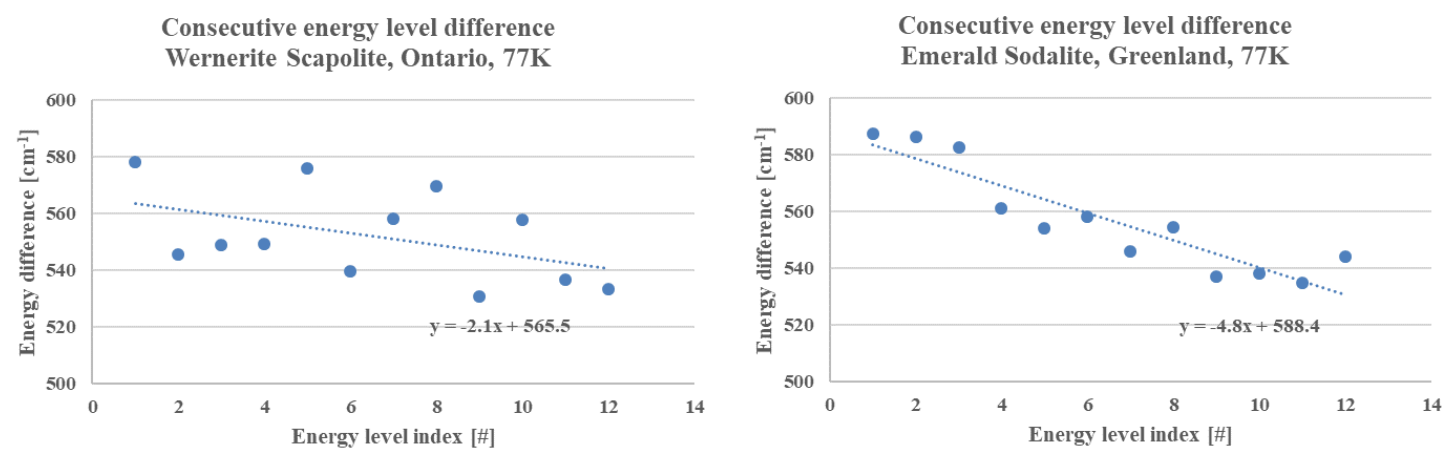

Figure 3. Energy difference for consecutive energy levels. Negative slope indicates a softening of the harmonic potential.

Other topics that can be addressed through this exercise are

1. Perturbation theory to fit the variation in the energy level differences and evaluate coefficients of higher order contributions to the potential,

2. Franck-Condon principle for calculating transition strengths and the overlap of the ground and excited state wavefunctions,

3. Huang-Rhys parameter, S, used to characterize the electron-vibrational coupling, and

4. Morse potential which is an adaptation of the QHO potential with 2 anharmonicity parameters.

The instructions with equipment list for the laboratory exercise appear at the end of this article.

\section{ACKNOWLEDGMENTS}

This laboratory exercise is based on work done by Sarah Friedensen ' 15 and Nancy Pruett '18, and was funded by the Davidson Research Initiative and Davidson College. The initial draft written by Henry Brooks '19. Edited and expanded by Dan Boye, Laurence Cain and Mario Belloni.

We wish to give special thanks to Mark Cole of www.naturesrainbows.com for his generous supply of mineral samples.

\section{REFERENCES}

[1] Airken Sidike, I. Kusachi, S. Kobayashi, K. Atobe, N. Yamashita, "Photoluminescence spectra of S2- center in natural and heat treated scapolites," Phys Chem Minerals 35:137-145 (2008).

[2] Airken Sidike, Alifu Sawuti, Xiang-Ming Wang, Heng-Jiang Zhu, S. Kobayashi, I. Kusachi, N. Yamashita, "Fine structure in photoluminescence spectrum of $\mathrm{S}_{2}{ }^{-}$center in sodalite," Phys Chem Minerals 34:477-484 (2007).

[3] D. Curie, B. Canny, P. Jaszczyn-Kopec, H.K. Liu, "The effects of hydrostatic and uniaxial pressures on vibronic spectra," J Lumin. 24/25:145-154 (1981). 


\section{The diatomic Sulfur anion $\left(\mathbf{S}_{2}\right)^{-}$modeled as a Quantum Harmonic Oscillator}

\section{OBJECTIVE:}

In this lab, you will study the energy levels of the diatomic sulfur anion $\left(\mathrm{S}_{2}\right)^{-}$contained within a mineral. Fluorescence emission spectra will be taken at room temperature and at $77 \mathrm{~K}$. The spectra will be analyzed to determine differences in energy among the vibrational energy states of the ground state potential well, the shape of the ground state well and other defining parameters of this system.

\section{BACKGROUND:}

Minerals are formed in the high temperature, high pressure environment within Earth's core and work their way to the surface through a variety of geophysical processes. Depending on the local mix of elements, different minerals can form simple to quite complex structures. Minerals can contain a fluorescence activator that, when the mineral is exposed to ultraviolet light, will glow brightly and colorfully. Nature's Rainbow (www.naturesrainbows.com) is a fluorescent mineral supersite! At the website Online Database of Luminescent Minerals (www.fluomin.org/uk/), information about minerals including names, colors and activators, can be searched. In minerals such as Wernerite (Ontario), emerald sodalite (Greenland), tugtupite (Greenland), among others, the activator is the diatomic sulfur anion $\left(\mathrm{S}_{2}\right)^{-}$. This anion is not part of the regular arrangement of ions that make up the mineral structure but is located within the vacant spaces of the mineral matrix.

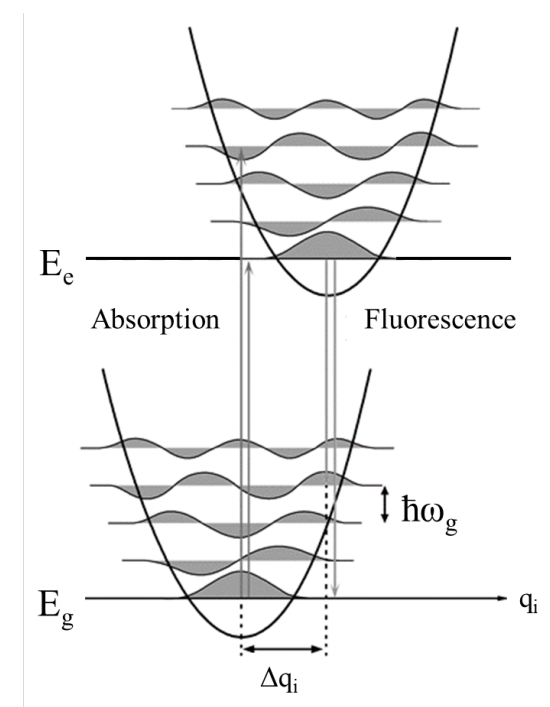

Figure 1. Ground and excited electronics levels plus vibrational levels coupled to the electronic levels.

A diatomic molecule can be modeled to a first approximation as a quantum simple harmonic oscillator. Classically, the two sulfur atoms, plus another electron, are bound by a linear restoring force. The motion of the two atoms can be reduced to that of a single mass oscillating about an equilibrium position in a quadratic potential. The solution to the time-independent Schrödinger equation for a quadratic potential yields energy levels that are equally-spaced and energy eigenstates (wave functions) based on the Hermite polynomials. In addition to there being discrete electronic transitions between electronic eigenstates, the diatomic anion can make transitions between vibrational eigenstates. Figure 1 shows 
two electronic eigenstates of energy $E_{0}$ and $E_{l}$ and their associated vibrational levels. The axes of the two wells are offset in the coordinate $q_{i}$ because the excited anion has a different size and shape compared to the anion in the ground state.

A laser emitting photons of sufficient energy will raise some of the $\left(\mathrm{S}_{2}\right)^{-}$anions to an excited electronic state through the absorption process. Then the $\left(\mathrm{S}_{2}\right)^{-}$ion will relax to the lowest-energy vibrational state within the excited electronic state through non-radiative processes. From this lowest excited energy level, the electrons will fall back to the ground state, emitting photons ("fluorescing") as they do so.

As the electrons return to their ground state, some vibrational energies will be more favored than others - those ground vibrational levels with wave functions that overlap substantially with the excited state vibrational levels will be more favored, while those with less overlap will be less favored. Because the electronic emission transitions originate from the same energy level, the spacing of peaks within the spectrum will then be the spacing of the vibrational energy levels of the $\left(\mathrm{S}_{2}\right)^{-}$anion $\left(\hbar \omega_{\mathrm{g}}\right.$ in the diagram).

In Procedure I, we will begin our analysis by assuming a quadratic form of for the ground state well, so the equation of this well will be $\mathrm{V}=1 / 2 m \omega_{\mathrm{g}}{ }^{2} x^{2}$. This would give a spacing between energy levels of $\hbar \omega_{\mathrm{g}}$. In reality, however, the quadratic approximation may not be entirely accurate, which would affect the shape of the ground state well and thus the spacing of the energy levels. In Procedure II, you will take a low temperature spectrum to determine a more accurate shape of the ground state well. Procedure III will add more precision by zooming in on the weaker peaks at the high and low energy transitions.

\section{INITIAL INFORMATION}

Your instructor will provide you the names of the minerals and their place of origin that you will be studying. Look up the mineral family called Scapolite and write a few sentences about the Scapolite family (chemical composition, unit cell dimensions, crystal shape, history, locations found, etc.) What are the names of the end members of the scapolite family? What is the difference between scapolite and sodalite?

\section{PROCEDURE I: Determining the Spring Constant of $\left(\mathbf{S}_{2}\right)^{-}$}

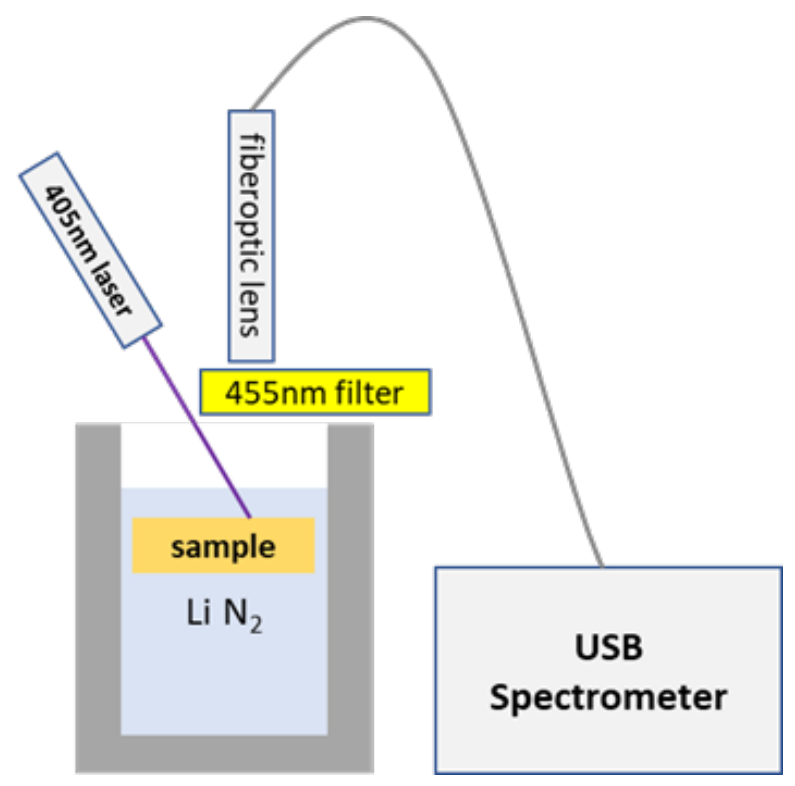

Figure 2. Experimental setup diagram. Computer connected to spectrometer is not shown. 


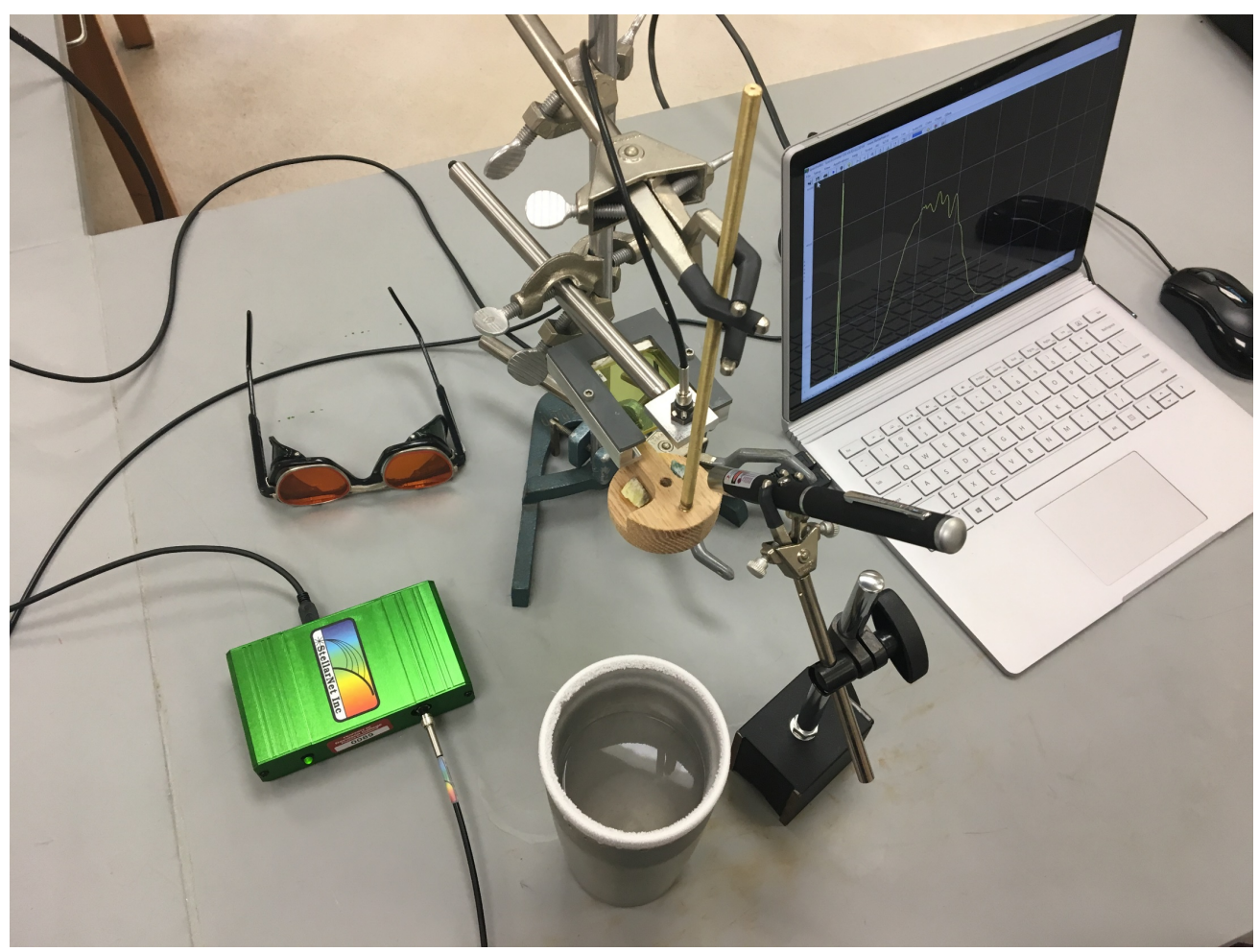

Figure 3. Photograph of experimental setup.

1. Set up the optical equipment as shown in Figures 2 and 3. The laser should hit the sample directly, and the filter and collecting lens should be aligned as straight as possible to maximize fluorescent light entering the fiber optic cable. Tighten all mechanical adjustments once the optical elements are properly aligned.

2. Open the spectrometer software and choose the scope mode.

3. Set the integration time so that the spectrum graph is not saturated (no wavelengths have the maximum signal) and set the acquisition to average several scans to reduce the effects of optical noise.

4. Turn off the laser and take a dark spectrum. Determine that the spectrometer software automatically subtracts the dark spectrum from subsequent spectra.

5. Turn on the laser and measure the fluorescence spectrum of the sample. Save the spectrum and open it in Excel. You may need to turn the Text to Columns. Plot the counts vs. wavelength spectrum in Excel. You should also create a column of photon energies, measured in $\mathrm{cm}^{-1}$ units, and plot counts vs. energy. Print both graphs for your report.

6. In Excel, determine the energies at which peaks occur, and from this data determine the average energy difference between peaks. This may be difficult because the peaks are not well-defined. Do the best you can.

7. From this average, determine the vibrational constant $k$, the spring constant for the coupled $\mathrm{S}$ atoms. $\omega_{g}=\sqrt{\frac{k}{\mu}}$, where $\mu=\frac{m_{S}}{2}$, and $\mathrm{m}_{\mathrm{S}}$ is the mass of a sulfur atom. What are the units of $k$ ? 


\section{PROCEDURE II: The Shape of the Ground State Well}

1. Fill the dewar $3 / 4$ full with liquid nitrogen, then clamp the dewar in place.

2. Lower the sample into the liquid nitrogen. The LiN2 will boil quickly at first, but by the time you are taking data, the boiling rate will have slowed so as not be a problem.

3. Adjust the optical elements as necessary to bring everything back into alignment.

4. Take a fluorescence spectrum as before, adjusting the integration time as necessary. If you change the integration time, take a new dark spectrum. Once you have obtained a clear spectrum, save the spectrum and plot it in Excel. Print the Excel graph for your report.

5. [Optional but higher quality analysis] Once you have obtained a clear spectrum, save the spectrum and plot it in Origin. Print the Origin graph for your report. In Origin, go to Analysis $\rightarrow$ Peaks and Baseline $\rightarrow$ Peak

Analyzer $\rightarrow$ Open Dialog.... The goal is to find the best positions of all the sub-peaks in your spectrum. In the top half of the dialog, check "Find Peaks." In the lower half, start with the auto find feature, then adjust parameters (clicking "Find" between each adjustment) until you have found satisfactory values for all the peaks.

6. Record the peak wavelengths and convert them to the units of energy, wavenumbers $\left[\mathrm{cm}^{-1}\right]$.

7. Create a plot of the difference in energy between consecutive peaks (in wavenumbers $\left[\mathrm{cm}^{-1}\right]$ ) vs. energy level index (each peak corresponds to a transition to a separate energy level; be sure that the transitions to lowest energy levels are on the left side of your plot). Find the linear best-fit for this data, then print the plot.

8. Based on the slope of the plot you just created, determine whether the ground state energy well is, in fact, parabolic, or whether it is steeper or shallower than a parabola at high energies (this change in shape is due to higher-order terms in the potential energy that we ignore with our initial approximation). Draw a qualitative sketch of this ground state energy well.

9. We say that an oscillator softens if the spring constant decreases and hardens if the spring constant increases. Does this well harden or soften with increasing energy?

\section{PROCEDURE III: Get a few more data points at the Highest and Lowest Energy Transitions}

1. With the same setup as in Procedure II, take one more spectrum at $77 \mathrm{~K}$, this time increasing the integration time so that the spectrometer is saturated in the mid-range, allowing us to see the less intense peaks on either side more clearly. As usual, plot in Excel, and print this graph for your report.

2. Use this spectrum to obtain more precise values for the peaks representing the higher and lower energy peaks.

3. With these new values, redo the plot of the difference in energy between consecutive peaks vs. energy level.

4. Print this plot, and comment on any observed changes from the plot obtained in Procedure II.

This lab exercise is based on work done by Sarah Friedensen '15 and Nancy Pruett '18, and was funded by the Davidson Research Initiative and Davidson College. Initial draft written by Henry Brooks '19. Edited and expanded by Dan Boye, Laurence Cain and Mario Belloni.

We wish to give special thanks to Mark Cole of www.naturesrainbows.com for his generous supply of mineral samples. 


\section{EQUIPMENT LIST}

USB Spectrometer

Color filter

Sample holder

Samples

Laser pointer

Laser safety goggles

Dewar
Shown is StellarNet GREEN Wave (350-1150nm range with $50 \mu \mathrm{m}$ slit) with $400 \mu \mathrm{m}$ fiber optic cable and collecting lens with holder (www.stellarnet.us)

Schott GG455nm longpass filter, 2 in. square with filter holder (also Thorlabs FGL455)

In-house design for two samples, made of oak

Miner Shop (www.minershop.com)

Ward's Science (www.wardsci.com)

$405 \mathrm{~nm}, 20-25 \mathrm{~mW}$

190-532nm \& 900-1700nm OD4+

(www.certified-laseryewear.com)

Shown is double-wall aluminum vacuum insulation coffee mug

Can use doubled Styrofoam cups

Can use silvered or unsilvered dewars (Pope Scientific Lab Grade)

www.popedewars.com/labgrade.html

\section{Assorted rods, clamps and stands}

\section{$S_{2}^{-}$ACTIVATOR MINERALS}

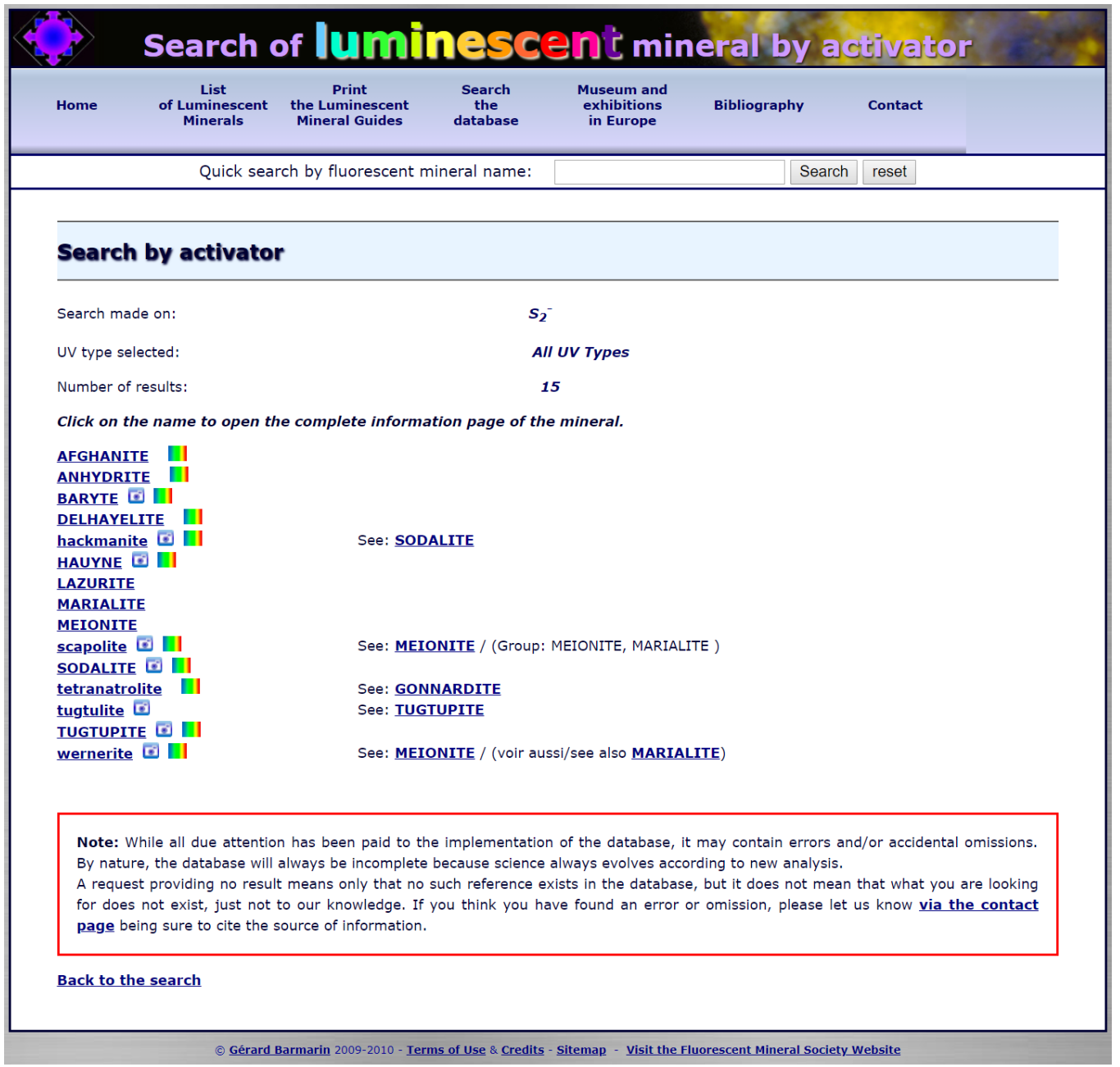

